\title{
Human papillomavirus infection in Bhutan at the moment of implementation of a national HPV vaccination programme
}

\author{
Ugyen Tshomo ${ }^{1}$, Silvia Franceschi' ${ }^{2}$, Dorji Dorji ${ }^{3}$, lacopo Baussano², Vanessa Tenet ${ }^{2}$, Peter JF Snijders ${ }^{4}$, \\ Chris JLM Meijer ${ }^{4}$, Maaike CG Bleeker ${ }^{4}$, Tarik Gheit ${ }^{2}$, Massimo Tommasino ${ }^{2}$ and Gary M Clifford ${ }^{2 *}$
}

\begin{abstract}
Background: Cervical cancer is the most common female cancer in Bhutan, the first low/middle-income country to implement a national human papillomavirus (HPV) vaccination programme.

Methods: To provide a robust baseline for future evaluations of vaccine effectiveness, cervical cell specimens were obtained from 2,505 women aged 18-69 years from the general population, and biopsies from 211 cervical intraepithelial neoplasia grade 3 (CIN3) and 112 invasive cervical cancer (ICC) cases. Samples were tested for HPV using GP5+/6+ PCR.

Results: Among the general population, HPV prevalence was 26\%, being highest (33\%) in women $\leq 24$ years, but remaining above 15\% in all age-groups. Determinants of HPV included age, marital status, and number of sexual partners. Among the eight percent with cytological abnormalities, 24 CIN3 and 4 ICC were histologically confirmed. Even after additional testing with a sensitive E7 PCR, no infections with vaccine-targeted HPV types were detected in the few vaccinated women $(n=34)$ compared to $6 \%$ prevalence in unvaccinated women of similar age $(p=0 \cdot 215)$.

Conclusion: Based upon type-specific prevalence among biopsies, at least 70\% of ICC in Bhutan are theoretically preventable by HPV16/18 vaccination, but screening programmes should be expanded among older women, who have an important underlying burden of CIN3 and ICC.
\end{abstract}

Keywords: Human papillomavirus, Prevalence, Cervical cancer, Bhutan

\section{Background}

In the Himalayan kingdom of Bhutan, cervical cancer represents the most common cancer among females, with an age-standardised incidence rate of approximately 13 cases per 100,000 person years [1], one of the highest in Asia. Given such a high burden of disease and the limited coverage of cervical screening, Bhutan became the first low- or middle-income country (LMIC) to initiate a national vaccination programme against human papillomavirus (HPV), the necessary cause of cervical cancer. In 2010, over 130,000 doses of quadrivalent (HPV16/18/6/11) HPV vaccine were administered, primarily at schools. The 3

\footnotetext{
* Correspondence: clifford@iarc.fr

${ }^{2}$ International Agency for Research on Cancer, 150 cours Albert Thomas, 69372 Lyon, cedex 08, France

Full list of author information is available at the end of the article
}

dose vaccination coverage is estimated at $92 \%$ among 12-18 year old girls [2]. Since 2011, 12-year old girls have continued to be vaccinated.

The impact of the vaccine programme on cervical cancer will not be seen for at least 20 years and, therefore, short-term evidence of vaccine programme effectiveness is crucial to encourage national planners to sustain HPV vaccination services. Hence, the Bhutan Ministry of Health, in collaboration with the International Agency for Research on Cancer (IARC), initiated a series of studies to evaluate the short term impact of HPV vaccine on HPV prevalence.

As no data existed on the burden of HPV among women in Bhutan, a baseline study of the prevalence of, and risk factors for, HPV infection prior to vaccination was initiated in 2011 according to the standardised protocol of the 
IARC HPV prevalence surveys [3]. Cervicovaginal samples were collected from women aged 18-69 years living in the capital, Thimphu, with a particular effort to oversample young ( $\leq 25$ years) women. In addition, biopsy/tissue specimens were collected from women diagnosed with cervical intraepithelial neoplasia grade 3 (CIN3) and invasive cervical cancer (ICC), for HPV detection and genotyping.

\section{Methods}

\section{General female population}

Between December 2011 and October 2012, a survey was conducted by the Ministry of Health of Bhutan in collaboration with the IARC, Lyon, France. The study aim was to enrol 2,500 women from the general population using an age-stratified approach, namely 1,000 women aged below 25 years old, 500 women aged 25 to 29 years old, 200 women in each five-year age group between 30-34 and 45-49 years, and 200 women aged $\geq 50$ years. Overrepresentation of young women was done in order to allow robust assessment of vaccine-induced changes in HPV prevalence in a repeat survey after 5 years. All mentally and physically competent women were eligible for the study, regardless of their marital status.

Study procedures were performed in the Reproductive Health Departments of Jigme Dorji Wangchuck National Referral Hospital (JDWNRH) in Thimphu, and in the hospital of Lungtenphu, a satellite town of Thimphu. To reach a broad and representative sample of the population living around the two hospitals, women were recruited in two ways: 1) Women residing in the pre-defined areas surrounding the two hospitals were visited at home by social workers and invited to join the study. Participation among the 1217 and 673 women invited from Thimphu and Lungtenphu was $25.7 \%$ and $54.2 \%$, respectively. 2) In addition, women consulting outpatient clinics in the two hospitals (mainly antenatal care or family planning clinics in which cervical cancer screening is also offered to women aged 20 or older), were also invited to join. Participation among women invited through the hospital was close to $100 \%$.

Some limited sociodemographic information was obtained from all women invited from home in Thimphu, among whom non-participants tended to be younger and more likely to be unmarried in comparison to participants. Following signature of an informed consent form, a structured questionnaire including information on socio-demographic characteristics, sexual behaviour of the women and of their partners, reproductive factors, use of contraceptive methods and smoking habits was administered to all study participants. History of HPV vaccination was asked to women aged $\leq 20$ years.

A cytobrush (Cervex-Brush, Rovers Medical Devices B.V., The Netherlands) was used for the collection of exfoliated cervical cells from the endocervix and ectocervix.
After preparation of a conventional Pap smear, the brush containing cellular material was placed in a vial containing PreservCyt medium (Cytyc, Boxbourough, MA, USA) and stored at $+4 \mathrm{C}$ until shipment.

Conventional Pap smears were read in the Department of Cytology, JDWNRH, and reported according to the 2001 Bethesda System [4]. All women with abnormal cervical findings were recalled to have a colposcope-aided examination and, if necessary, a colposcopically-directed biopsy and appropriate treatment by local gynaecologists. Histological confirmation of cervical tissue was performed at JDWNRH.

\section{Women with CIN3 and ICC}

Formalin-fixed paraffin-embedded biopsies were retrieved from all cases of ICC and CIN3 diagnosed between 2010 and 2012 at JDWNRH, Thimphu or the Eastern Regional Referral Hospital, Mongar. Biopsies were sent to the VU University Medical Center, Amsterdam, for HPV DNA testing and review of histology. After exclusion of 11 biopsies negative for beta-globin PCR and 177 biopsies without any histological evidence of CIN3 or cancer, 211 CIN3 and 112 ICC cases were included in following analyses (of which 17 CIN3 and 4 ICC, respectively, derived from women included in the general population survey described above).

\section{HPV testing and genotyping}

HPV testing was performed on all exfoliated cervical cells and biopsies in the Department of Pathology at the VU University Medical Center, Amsterdam. DNA was extracted from the PreservCyt sample using magnetic beads (Macherey-Nagel) on a robotic system (Hamilton Robotics) according to the manufacturer's instructions. Biopsies were sectioned using a 'sandwich' approach, whereby inner tumour sections were destined for HPV testing and outer sections for histological confirmation of tumour tissue (see above). One or more sections representing approximately $1 \mathrm{~cm}^{2}$ of tissue were predigested with proteinase $\mathrm{K}$ after which DNA was extracted using magnetic beads (Macherey-Nagel).

Beta-Globin PCR analysis was conducted first to confirm the presence of human DNA in all specimens [5]. The presence of HPV DNA was determined by conducting a general primer GP5+/6+- mediated PCR, which permits the detection of a broad spectrum of genital HPV types [6]. HPV positivity was assessed by hybridization of PCR products in an enzyme immunoassay with 2 oligoprobe cocktails that, together, detect the following 44 mucosal HPV types: HPV6, 11, 16, 18, 26, 30, 31, 32, 33, 34, 35, 39, $40,42,43,44,45,51,52,53,54,55,56,57,58,59,61,64$, $66,67,68,69,70,71,72,73,81,82,83,84,85,86,89$, and 90. Subsequent HPV genotyping was conducted by reverse-line blot hybridization of GP5+/6+ PCR products 
[7]. HPV types considered high-risk (HR) types for this analysis comprised HPV16, 18, 31, 33, 35, 39, 45, 51, 52, 56, 58, 59, and 68 [8]; possible HR types comprised HPV26, 30, 34, 53, 66, 67, 69, 70, 73, 82, and 85; all other HPV types were considered low-risk (LR).

DNA extracted from cervical cells of women aged $\leq$ 24 years, was additionally tested for the $13 \mathrm{HR}$ types plus HPV6 and 11 using an alternative PCR-based assay based upon multiplex E7 gene PCR and DNA microarray [9] at IARC. This assay has been validated against a WHO HPV LabNet proficiency panel [10], and is known to be more sensitive than GP5+/6+-mediated PCR in detecting low copy numbers of viruses, particularly in the presence of multiple infections [11].

\section{Statistical analyses}

In addition to crude prevalence, age-standardised HPV prevalence was computed using the world standardised population to allow comparisons of HPV prevalence with other IARC surveys [3]. Prevalence ratios (PR) for HPV positivity and corresponding 95\% confidence intervals (CI) were computed using two binomial regression models with a log link, the first adjusted for the study design variables age group $(<24 ; 25-29,30-34,35-39,40-44$, and $>45$ years $)$ and hospital where examined (JDWNRH or Lungthenphu), and a second model that adjusted additionally for lifetime sexual partners. Risk trends were assessed by considering categories as continuous variables. PRs were also used to compare HPV type-specific prevalence in HPV-positive ICC and normal cytology.

\section{Ethical approval}

The present study had the approval of both the Research Ethical Board of the Bhutan Ministry of Health and the IARC Ethics Committee.

\section{Results}

\section{General female population}

Of the 2,525 participants who provided cervical cell samples, 2,505 had valid HPV results and were included in the following analyses, including 1,638 and 867 recruited at JDWNRH and Lungthenphu hospitals, respectively. Among the 2,468 women with a valid PAP smear result, $196(8 \%)$ had an abnormal cytological diagnosis, including 123 (58\%) with atypical squamous cells of undetermined significance, atypical squamous cells cannot exclude high-grade lesion or atypical glandular cells of undetermined significance (ASCUS/ASC-H/AGUS), 59 (2\%) low-grade squamous intraepithelial lesions (LSIL), and 14 (1\%) high-grade squamous intraepithelial lesions or worse (HSIL+). Histological confirmation was obtained for 135 women with abnormal cytology, among whom 23 CIN2, 24 CIN3 and 4 ICC were histologically confirmed.
Overall HPV prevalence was 26\% (95\% CIs: 24-28), being 23\% (95\% CIs: 21-25) among women with normal cytology (Table 1). Corresponding overall HPV prevalence age-standardised to the world population was $27 \%$. The prevalence of HPV types in abnormal cytology was $61 \%$ (Table 1), being 52\%, 81\%, and 57\% in ASCUS/ASC-H/ AGUS, LSIL and HSIL+, respectively. In total, 437 (17\%) women had single-type and 218 (9\%) had multiple-type infections. Crude and age-standardised prevalence of HR types was $18 \%$. Crude prevalence of possible HR HPV was 5\%. HPV16 was the most common HPV type both in women with normal (3\%) and abnormal (14\%) cytology.

Figure 1 shows the age-specific prevalence of HPV and cytological abnormalities, classified hierarchically into (1) HPV16 or 18, (2) other HR types and (3) LR types only. Overall HPV prevalence was highest (33\%, 95\% CI: 30-36) among women $<24$ years, and decreased with age, down to $16 \%$ (95\% CI: $11-22$ ) among women aged $\geq 50$ years. Prevalence of cytological abnormalities varied between $4 \%$ in women aged $\geq 50$ years and $11 \%$ in women aged $40-44$ years. The prevalence of CIN3 or worse was about $1 \%$ in all age-groups, but all 4 ICC were diagnosed only in women aged over 35 years old (data not shown).

Table 2 shows the relationship between HPV positivity and various characteristics of participants. In a model adjusted for age and hospital only, significant determinants of HPV positivity included age group (PR for $\geq 45$ vs $\leq 24$ years $=0.53 ; 95 \% \mathrm{CI}: 0 \cdot 42-0 \cdot 69)$, hospital (PR for Lungthenphu versus JDWNRH = 0 - 81; 95\% CI: 0 -70-0 • 94), ethnic group (PR for Lhotsampa vs Scharchop $=0.69$; 95\% CI: 0.57-0.83), marital status (PR for separated/ widowed versus married $=1 \cdot 34 ; 95 \% \mathrm{CI}: 1 \cdot 04-1 \cdot 74)$, number of marriages (PR for $\geq 2$ vs $1=1 \cdot 62$; 95\% CI: 1 . 32-1.99), number of lifetime sexual partners ( $P R$ for $\geq 3$ vs $1=1 \cdot 93$; 95\% CI: $1 \cdot 21-3.08)$, number of pregnancies (PR for nulliparous vs $1=1 \cdot 46$; 95\% CI: $1 \cdot 23-1 \cdot 74$ ), age at first pregnancy (PR for $\leq 22$ vs $<19=0 \cdot 78$; $95 \% \mathrm{CI}$ : 0.63-0.95), husbands extramarital relations (PR for Yes, during marriage $=1.71$, 95\% CI 1.27-2.31), use of any contraceptive method (PR for ever vs never $=0.80$; 95\% CI: $0 \cdot 69-0 \cdot 93)$, history of PAP smear ( $\mathrm{PR}=0 \cdot 77$; 95\% CI: 0 -66-0 -90), receiving cash for sex $(\mathrm{PR}=2 \cdot 77 ; 95 \%$ CI: $1 \cdot 55-4 \cdot 95)$ or having genital warts $(\mathrm{PR}=2 \cdot 17 ; 95 \%$ CI: $1 \cdot 06-4 \cdot 45)$. All these associations remained significant in a second model additionally adjusted for lifetime number of sexual partners, with the exception of number of marriages.

No associations with HPV positivity were seen for education level (49\% of women reported no formal education), source of recruitment (27\% from home, $73 \%$ from hospital) (Table 2), family income, smoking ( $11 \%$ ever smokers), chewing of non-tobacco products (36\% ever chewers), occupation (67\% housewife), age at menarche (median = 14 years), age at first sexual intercourse (median $=19$ years), 
Table 1 Prevalence of human papillomavirus (HPV) types by cytological findings and overall among 2,505 women in Bhutan, 2011-2012

\begin{tabular}{|c|c|c|c|c|c|c|c|c|c|c|c|c|}
\hline \multirow[t]{2}{*}{ HPV type } & \multicolumn{4}{|c|}{ Normal $(n=2272)$} & \multicolumn{4}{|c|}{ Abnormal $(n=196)$} & \multicolumn{4}{|c|}{ Total $(n=2505)^{a}$} \\
\hline & Single & Multiple & Total & $(\%)$ & Single & Multiple & Total & $(\%)$ & Single & Multiple & Total & (\%) \\
\hline HPV- & - & - & 1750 & $(77)$ & - & - & 76 & (39) & - & - & 1850 & (74) \\
\hline $\mathrm{HPV}+$ & 358 & 164 & 522 & (23) & 70 & 50 & 120 & $(61)$ & 437 & 218 & 655 & (26) \\
\hline \multicolumn{13}{|l|}{ High-risk } \\
\hline 16 & 48 & 30 & 78 & (3.4) & 15 & 13 & 28 & (14) & 65 & 44 & 109 & $(4.4)$ \\
\hline 18 & 26 & 27 & 53 & $(2.3)$ & 2 & 9 & 11 & (5.6) & 29 & 37 & 66 & (2.6) \\
\hline 31 & 14 & 7 & 21 & $(0.9)$ & 4 & 3 & 7 & (3.6) & 19 & 10 & 29 & $(1.2)$ \\
\hline 33 & 9 & 12 & 21 & $(0.9)$ & 6 & 4 & 10 & $(5.1)$ & 16 & 17 & 33 & (1.3) \\
\hline 35 & 3 & 6 & 9 & $(0.4)$ & 3 & 2 & 5 & (2.6) & 6 & 8 & 14 & $(0.6)$ \\
\hline 39 & 11 & 5 & 16 & $(0.7)$ & 0 & 6 & 6 & (3.1) & 11 & 11 & 22 & $(0.9)$ \\
\hline 45 & 9 & 19 & 28 & $(1.2)$ & 1 & 8 & 9 & (4.6) & 10 & 27 & 37 & (1.5) \\
\hline 51 & 15 & 14 & 29 & $(1.3)$ & 6 & 8 & 14 & (7.1) & 21 & 22 & 43 & (1.7) \\
\hline 52 & 16 & 12 & 28 & $(1.2)$ & 3 & 3 & 6 & (3.1) & 19 & 15 & 34 & (1.4) \\
\hline 56 & 17 & 14 & 31 & (1.4) & 4 & 8 & 12 & (6.1) & 21 & 23 & 44 & (1.8) \\
\hline 58 & 14 & 13 & 27 & $(1.2)$ & 11 & 6 & 17 & (8.7) & 25 & 19 & 44 & (1.8) \\
\hline 59 & 21 & 19 & 40 & $(1.8)$ & 4 & 2 & 6 & (3.1) & 26 & 21 & 47 & (1.9) \\
\hline 68 & 4 & 5 & 9 & $(0.4)$ & 3 & 1 & 4 & $(2.0)$ & 7 & 6 & 13 & $(0.5)$ \\
\hline Any & $214^{b}$ & 125 & $339^{\mathrm{b}}$ & (15) & 62 & 42 & 104 & (53) & $282^{b}$ & 169 & $451^{b}$ & (18) \\
\hline
\end{tabular}

\section{Possibly high-risk}

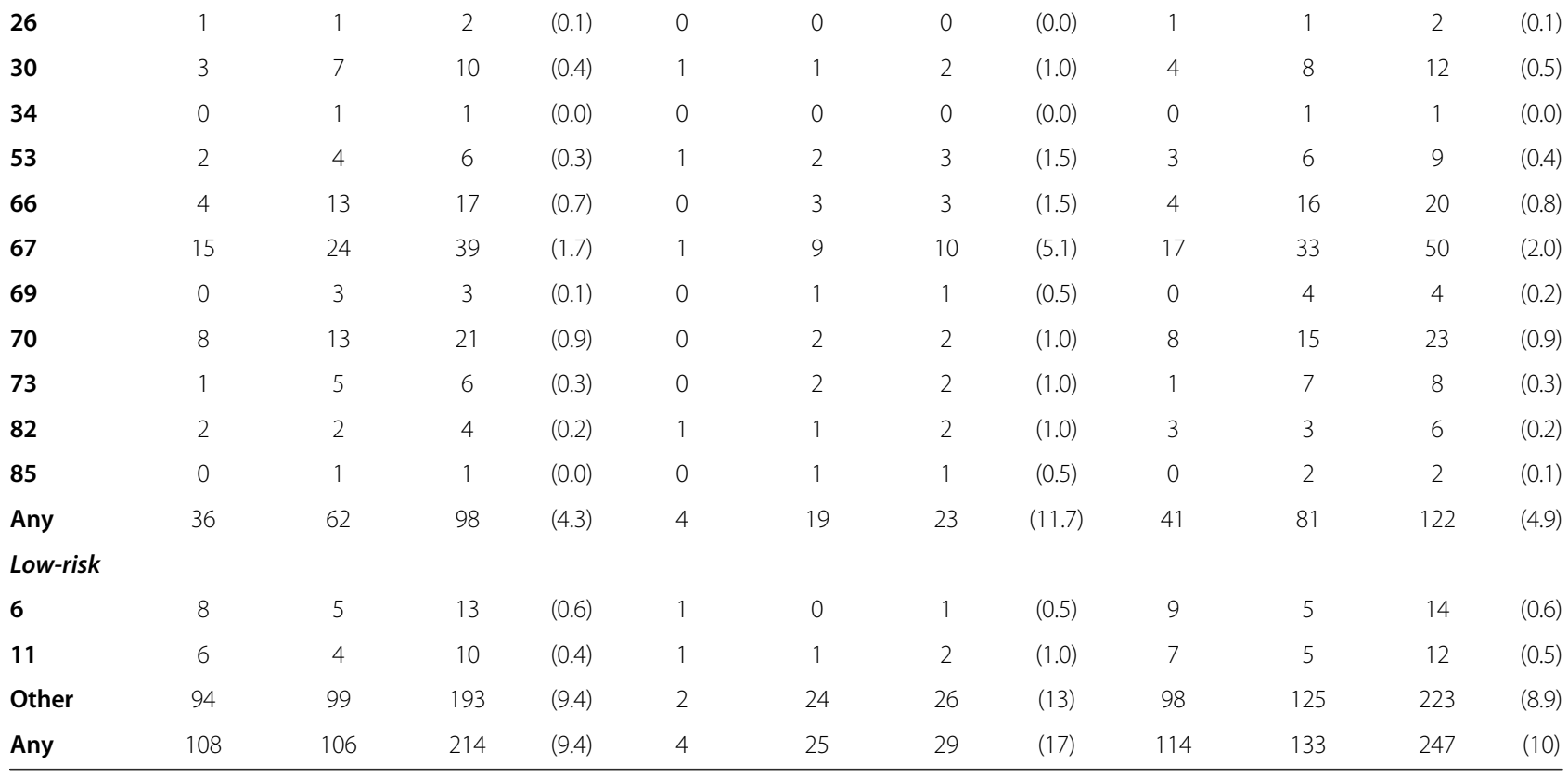

a 37 inadequate cytology are also included in the total.

${ }^{\mathrm{b}}$ The total is not equal to the sum of the 13 high-risk because of 7 uncharacterized high-risk infections that are also included in the total.

and difference in age with partner at first sexual intercourse (median $=4$ years) (data not shown).

Upon additional testing of women aged $\leq 24$ years with multiplex E7 gene PCR (Figure 2), an additional 27 (3\%) and 79 (9\%) women became positive for vaccine-targeted HPV types and non-vaccine-targeted HR HPV types, respectively. Very few GP5+/6+ positive women tested negative by multiplex E7 PCR (2 and 13 for vaccinetargeted HPV types and non-vaccinated HR HPV types, respectively, data not shown). A small number of women aged $\leq 20$ years reported HPV vaccination $(n=34)$, among whom the prevalence of non-vaccine targeted HR HPV 


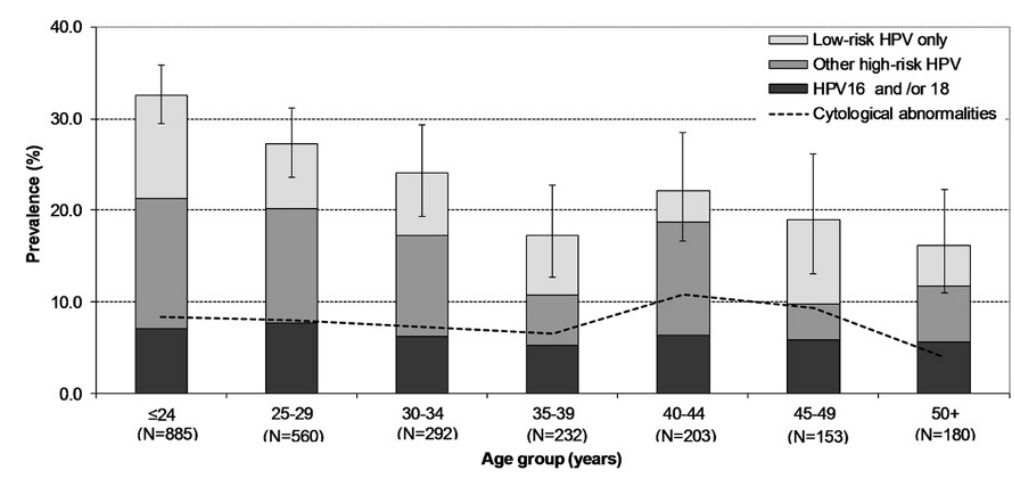

Figure 1 Age-specific prevalence of human papillomavirus (HPV) DNA and of cytological abnormalities among 2,505 women. Bhutan, 2011-2012. HPV = human papillomavirus.

types $(12 \%)$ was similar to that seen in unvaccinated women of the same age, and that in women aged 21-24 (Figure 2). However, no infections with vaccine-targeted HPV types were detected in vaccinated women, compared to $6 \%$ in unvaccinated women of the same age (2-sided Fisher's exact test, $P=0 \cdot 215)$, and $9 \%$ in women aged $21-24$ $(\mathrm{p}=0 \cdot 064)$.

\section{Women with CIN3 and ICC}

Type-specific HPV prevalence is described in Table 3 for biopsies of 211 CIN3 and 112 ICC cases of which $87 \%$ and $88 \%$, respectively, were HPV-positive. Type-specific HPV prevalence is reported among HPV-positive cases only and is compared to that among $523 \mathrm{HPV}$-positive women with normal cytology from the general population survey (Table 3). HPV16 was by far the most common HPV type, being detected in $60 \%$ and $63 \%$ of HPV-positive CIN3 and ICC, respectively. HPV16/18 and HPV16/18/31/33/45/52/ 58 together accounted for $70 \%$ and $88 \%$ of ICC, respectively. Upon comparison with HPV-positive women with normal cytology, PRs for women with ICC were 6.5 (95\% CI: 4.5-9.4) for HPV16 and 6.7 (95\% CI: 3.8-12.1) for HPV16/18/31/33/45/52/58, but did not exceed one for any other HR HPV types. Possible HR, LR and multipletype infections were also much less common in ICC than in HPV-positive women with normal cytology (Table 3). HPV18 and HPV45 were detected in a larger proportion of ICC than CIN3, whereas the opposite was true for HPV58.

\section{Discussion}

These data provide a picture of HPV infection in the adult female population of Bhutan just after the country embarked upon a highly successful national programme of HPV vaccination among 12-18 year old girls, to serve as a baseline for future impact monitoring. Data represent that of an almost entirely vaccine-free population and disclose a relatively high prevalence of HR HPV infection (18\%), particularly among women younger than
25 years (22\%). Findings can be confidently compared with other IARC HPV surveys that were conducted according to a similar sampling and HPV testing protocol. In this respect, the age-standardised HR HPV prevalence in Bhutan (18\%) ranks below that of Guinea (31\%) [12] and Mongolia (25\%) [13], but is similar to the prevalence detected in Nigeria (18\%) [14] and Vanuatu (19\%) [15]. It is more elevated than that found in many other areas at high-risk for cervical cancer in Asia, including that in neighbouring countries of India (12\%) [16], China (12-13\%) [17-19] and Nepal (6\%) [20]. HR HPV prevalence is strongly correlated internationally with cervical cancer incidence rates [21] and the high prevalence of cervical carcinoma ( 1 out of every 200 women aged $35+$ years) in our study population is consistent with recent estimates of cervical cancer incidence in Bhutan [1].

HPV16 was by far the most frequently detected type in Bhutan, increasing from $3 \%$ of normal cytology up to $63 \%$ of HPV-positive ICC. Such a high proportion of HPV16 in cervical cancer agrees with findings of a meta-analysis in which the proportion of HPV16-positive ICC in Western/ Central Asia (66\%) was the highest among all world regions [19]. Infections with vaccine types HPV16 and/or 18 accounted for $24 \%$ of HR HPV positivity in the general female population and for $70 \%$ of cervical cancer, which gives an estimate of the potential future impact of the vaccine programme, if one simply assumes $100 \%$ efficacy and zero cross-protection against non-vaccine types. The proportion of ICC theoretically preventable by the seven HR HPV types (HPV16/18/31/33/45/52/58) included in a future nonavalent vaccine is $88 \%$. Of note, HPV18 and HPV45 were under-represented in CIN3 in comparison to cancer, whereas certain HPV types, particularly HPV58, were responsible for a much larger proportion of CIN3 than of ICC. These mirror findings from other world regions in a recent meta-analysis [22].

Risk factors associated with cervical HPV infection were largely consistent with previous IARC HPV Surveys. Lifetime number of sexual partners was an important 
Table 2 Prevalence ratios (PR) for human papillomavirus (HPV) positivity and corresponding $95 \%$ confidence intervals (Cls) according to selected characteristics among 2,505 women in Bhutan, 2011-2012

\begin{tabular}{|c|c|c|c|c|c|c|}
\hline Characteristic & $\mathrm{N}$ women ${ }^{\mathrm{a}}$ & HPV-positive (\%) & Adjusted $^{\mathrm{b}}$ PR & $95 \% \mathrm{Cl}$ & Adjusted $^{\mathrm{C}} \mathrm{PR}$ & $95 \% \mathrm{Cl}$ \\
\hline \multicolumn{7}{|l|}{ Age-group (years) } \\
\hline$\leq 24$ & 885 & $289(32.7)$ & 1 & - & 1 & - \\
\hline $25-34$ & 852 & $223(26.2)$ & 0.82 & $0.71-0.95$ & 0.81 & $0.70-0.94$ \\
\hline $35-44$ & 435 & $85(19.5)$ & 0.61 & $0.49-0.75$ & 0.57 & $0.46-0.71$ \\
\hline$\geq 45$ & 333 & $58(17.4)$ & 0.53 & $0.42-0.69$ & 0.51 & $0.39-0.65$ \\
\hline$x_{1}^{2}$ for trend & & & $p<0.001$ & & $p<0.001$ & \\
\hline \multicolumn{7}{|c|}{ Hospital where examined } \\
\hline Thimphu & 1638 & $460(28.1)$ & 1 & - & 1 & - \\
\hline Lungthenphu & 867 & $195(22.5)$ & 0.81 & $0.70-0.94$ & 0.82 & $0.71-0.95$ \\
\hline \multicolumn{7}{|l|}{ Type of invitation } \\
\hline Clinic & 1835 & $483(26.3)$ & 1 & - & 1 & - \\
\hline Door to door & 670 & $172(25.7)$ & 1.06 & $0.91-1.24$ & 1.08 & $0.92-1.26$ \\
\hline \multicolumn{7}{|l|}{ Ethnic group } \\
\hline Scharchop & 949 & $264(27.8)$ & 1 & - & 1 & - \\
\hline Ngalop & 586 & $180(30.7)$ & 1.11 & $0.95-1.30$ & 1.11 & $0.94-1.30$ \\
\hline Lhotsampa & 585 & $116(19.8)$ & 0.69 & $0.57-0.83$ & 0.70 & $0.57-0.85$ \\
\hline Other & 371 & $88(23.7)$ & 0.90 & $0.74-1.11$ & 0.89 & $0.72-1.09$ \\
\hline \multicolumn{7}{|l|}{ Education (yrs) } \\
\hline Illiterate & 1209 & $292(24.2)$ & 1 & - & 1 & - \\
\hline $1-9$ & 512 & $132(25.8)$ & 1.02 & $0.85-1.21$ & 1.02 & $0.85-1.21$ \\
\hline$\geq 10$ & 750 & $221(29.5)$ & 1.07 & $0.92-1.24$ & 1.09 & $0.94-1.28$ \\
\hline$x_{1}^{2}$ for trend & & & $p=0.415$ & & $p=0.274$ & \\
\hline \multicolumn{7}{|l|}{ Marital status } \\
\hline Married & 2298 & $586(25.5)$ & 1 & - & 1 & - \\
\hline Single & 54 & $19(35.2)$ & 1.22 & $0.84-1.76$ & 1.60 & $1.14-2.24$ \\
\hline Separated/widowed & 137 & $42(30.7)$ & 1.34 & $1.04-1.74$ & 1.31 & $1.01-1.69$ \\
\hline \multicolumn{7}{|c|}{ Number of marriages $^{d}$} \\
\hline 1 & 2209 & $549(24.9)$ & 1 & - & 1 & - \\
\hline$\geq 2$ & 201 & $67(33.3)$ & 1.62 & $1.32-1.99$ & 1.27 & $0.86-1.86$ \\
\hline \multicolumn{7}{|c|}{ Number of pregnancies } \\
\hline Nulliparous & 276 & $117(42.4)$ & 1.46 & $1.23-1.74$ & 1.50 & $1.26-1.78$ \\
\hline 1 & 776 & $228(29.4)$ & 1 & - & 1 & - \\
\hline 2 & 588 & $134(22.8)$ & 0.85 & $0.70-1.03$ & 0.83 & $0.68-1.01$ \\
\hline$\geq 3$ & 839 & $163(19.4)$ & 0.84 & $0.67-1.05$ & 0.81 & $0.65-1.01$ \\
\hline$x_{1}^{2}$ for trend $d^{e}$ & & & $p=0.159$ & & $p=0.090$ & \\
\hline \multicolumn{7}{|c|}{ Age at first pregnancy } \\
\hline$<19$ & 554 & $145(26.2)$ & 1 & - & 1 & - \\
\hline $19-21$ & 885 & $222(25.1)$ & 0.93 & $0.78-1.11$ & 0.98 & $0.82-1.17$ \\
\hline$\geq 22$ & 754 & $155(20.6)$ & 0.78 & $0.63-0.95$ & 0.81 & $0.66-0.99$ \\
\hline$x_{1}^{2}$ for trend & & & $p=0.014$ & & $p=0.041$ & \\
\hline \multicolumn{7}{|l|}{ Contraceptive use } \\
\hline Never & 562 & $173(30.8)$ & 1 & - & 1 & - \\
\hline Ever & 1860 & $458(24.6)$ & 0.80 & $0.69-0.93$ & 0.76 & $0.66-0.89$ \\
\hline
\end{tabular}


Table 2 Prevalence ratios (PR) for human papillomavirus (HPV) positivity and corresponding $95 \%$ confidence intervals (Cls) according to selected characteristics among 2,505 women in Bhutan, 2011-2012 (Continued)

\begin{tabular}{|c|c|c|c|c|c|c|}
\hline Hormonal only & 853 & $233(27.3)$ & 0.82 & $0.69-0.96$ & 0.78 & $0.66-0.92$ \\
\hline Hormonal + other & 276 & $63(22.8)$ & 0.79 & $0.61-1.02$ & 0.74 & $0.57-0.95$ \\
\hline Other only & 777 & $174(22.4)$ & 0.78 & $0.65-0.94$ & 0.75 & $0.63-0.90$ \\
\hline \multicolumn{7}{|l|}{ Lifetime sexual partners ${ }^{f}$} \\
\hline 1 & 2228 & $566(25.4)$ & 1 & - & 1 & - \\
\hline 2 & 180 & $60(33.3)$ & 1.54 & $1.24-1.92$ & 1.54 & $1.24-1.92$ \\
\hline$\geq 3$ & 26 & $10(38.5)$ & 1.93 & $1.21-3.08$ & 1.93 & $1.21-3.08$ \\
\hline$x_{1}^{2}$ for trend & & & $p<0.001$ & & $p<0.001$ & \\
\hline \multicolumn{7}{|c|}{ Husbands "extramarital" sexual relationships ${ }^{d}$} \\
\hline No & 1966 & $481(24.5)$ & 1 & - & 1 & - \\
\hline Yes, before marriage only & 308 & $95(30.8)$ & 1.30 & $1.08-1.55$ & 1.23 & $1.02-1.48$ \\
\hline Yes, during marriage & 78 & $28(35.9)$ & 1.71 & $1.27-2.31$ & 1.49 & $1.09-2.05$ \\
\hline Uncertain & 76 & $22(29.0)$ & 1.02 & $0.72-1.47$ & 1.00 & $0.70-1.43$ \\
\hline \multicolumn{7}{|c|}{ History of receiving cash for sex ${ }^{g}$} \\
\hline No & 2467 & $641(26.0)$ & 1 & - & 1 & - \\
\hline Yes & 9 & $5(55.6)$ & 2.77 & $1.55-4.95$ & - & - \\
\hline \multicolumn{7}{|l|}{ Last PAP smear (yrs) } \\
\hline Never & 1367 & $418(30.6)$ & 1 & - & 1 & - \\
\hline Ever & 1114 & $229(20.6)$ & 0.77 & $0.66-0.90$ & 0.77 & $0.66-0.90$ \\
\hline \multicolumn{7}{|c|}{ Evidence of genital warts at visit ${ }^{\mathrm{h}}$} \\
\hline No & 2473 & $640(25.9)$ & 1 & - & 1 & - \\
\hline Yes & 5 & $3(60.0)$ & 2.17 & $1.06-4.45$ & 2.23 & $1.09-4.59$ \\
\hline
\end{tabular}

${ }^{\text {a }}$ Some figures do not add up to the total due to missing values.

${ }^{\mathrm{b}}$ Adjusted for age and hospital as appropriate.

${ }^{\mathrm{C}}$ Adjusted for age, hospital and lifetime sexual partners as appropriate.

${ }^{d}$ Among ever married women only.

eAmong parous women only.

$f_{3}$ virgins excluded.

${ }^{9}$ Model does not converge for PR adjusted for lifetime sexual partners.

${ }^{\mathrm{h}} \mathrm{PR}$ not adjusted for age because model does not converge.

determinant of HPV positivity, although the mean reported number of women's lifetime sexual partners (1.1) was relatively low. Being single, separated or divorced, as well as a history of receiving cash for sex (albeit rarely reported), were also positively associated with HPV prevalence. Nulliparous women had a significantly higher risk of being HPV-positive, even after adjustment, in agreement with a pooled analysis of previous IARC HPV Prevalence Surveys [23], but the linear trend by number of pregnancies was not significant among parous women. Use of both hormonal and non-hormonal contraceptives and condoms were associated with lower HPV prevalence in Bhutan, in contrast to previous findings of pooled analyses of the IARC HPV prevalence surveys that found no associations $[23,24]$. However, the effect of residual confounding by under-reported high-risk sexual behaviour on certain associations cannot be ruled out. Lastly, a lower prevalence (20\%) was also remarked among the Lhotsampa ethnic group, who are an ethnically Nepalese and largely
Hindu population. However, this prevalence remains somewhat higher than that observed in the IARC HPV prevalence survey in Nepal (9\%) [20].

Forty-five percent of study participants reported to having a prior PAP smear, which gives an estimation of the coverage of the cytology-based programme that has been targeting women living in Thimphu since 1999. Cervical screening is unavailable, however, in most rural and higher-altitude areas of Bhutan. One possibility to improve nationwide coverage is the application of an HPV testing approach, e.g. careHPV in women aged 30 or older. Our data suggest that approximately $15 \%$ of screened women aged 30 or older might test positive for HR HPV and require diagnostic follow-up and treatment.

This study has weaknesses and strengths. In particular, the extent to which our study population is representative of the general female population in Thimphu, and Bhutan as a whole, is difficult to evaluate. However, HPV infection is asymptomatic and self-selection of 


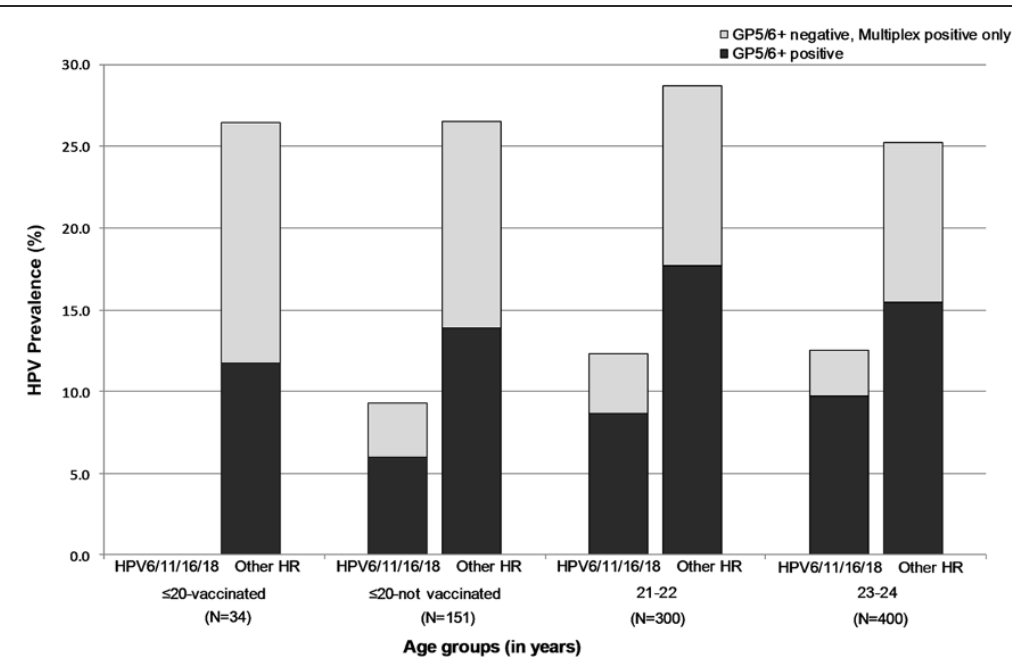

Figure 2 Age-specific prevalence of human papillomavirus (HPV) DNA among 885 women $\leq 24$ years old, according to two HPV DNA tests (GP5+/6+ and multiplex E7). Bhutan, 2011-2012. HPV = human papillomavirus; HR = high-risk.

women by level of HPV risk is unlikely to have occurred. Furthermore, there was no significant difference in HPV prevalence by whether women were recruited through hospital consultations or from their homes, neither was there any substantial difference in their distribution of any of the study variables in Table 1.

Strengths include the relatively large number of women, especially those younger than 25 years, and the high rate of colposcopical follow-up and histological confirmation for women with abnormal cytology, allowing a strong gold standard of disease ascertainment. Furthermore, we used two high-quality PCR tests of which one, GP5+/6+, is clinically validated and conducted in a central laboratory that allows comparability with other studies, notably previous IARC HPV Surveys.

We made a particular effort to sample a large number of women aged $\leq 24$ years, and to test with a second highlysensitive E7 multiplex PCR assay [9]. As expected, adding this test approximately doubled the HR HPV prevalence over that detected by GP5+/6+ [11]. The greater relative

Table 3 Prevalence of selected human papillomavirus (HPV) types and groups of HPV types among HPV-positive women, by cervical status

\begin{tabular}{|c|c|c|c|c|c|c|c|}
\hline \multirow[t]{2}{*}{ HPV type } & \multicolumn{2}{|r|}{$\begin{array}{l}\text { Normal }^{\mathrm{a}} \\
(\mathrm{N}=2272)\end{array}$} & \multicolumn{2}{|r|}{$\begin{array}{c}\mathrm{CIN}^{\mathrm{b}} \\
(\mathrm{N}=211)\end{array}$} & \multicolumn{2}{|r|}{$\begin{array}{l}\text { Cancer }^{\mathrm{b}} \\
(\mathrm{N}=112)\end{array}$} & \multirow[t]{2}{*}{$\begin{array}{l}\text { Cancer: normal cytology } \\
\text { Prevalence ratio }(95 \% \mathrm{Cl})\end{array}$} \\
\hline & $n$ & (\% of HPV pos) & n & (\% of HPV pos) & $n$ & (\% of HPV pos) & \\
\hline Any & 522 & - & 183 & - & 99 & - & - \\
\hline 16 & 78 & (15) & 109 & $(60)$ & 62 & (63) & $5.8(4.0-8.3)$ \\
\hline 18 & 53 & (10) & 4 & $(2)$ & 7 & (7) & $0.7(0.4-1.5)$ \\
\hline $16 / 18$ & 127 & $(24)$ & 113 & (62) & 69 & (70) & $5.0(3.4-7.4)$ \\
\hline 31 & 21 & (4) & 14 & (8) & 4 & (4) & $1.0(0.4-2.5)$ \\
\hline 33 & 21 & (4) & 12 & (7) & 2 & $(2)$ & $0.5(0.1-2.0)$ \\
\hline 45 & 28 & (5) & 2 & (1) & 5 & (5) & $0.9(0.4-2.2)$ \\
\hline 52 & 28 & (5) & 2 & (1) & 3 & (3) & $0.6(0.2-1.8)$ \\
\hline 58 & 27 & (5) & 32 & (18) & 5 & (5) & $1.0(0.4-2.2)$ \\
\hline $16 / 18 / 31 / 33 / 45 / 52 / 58$ & 235 & $(45)$ & 168 & $(92)$ & 87 & (88) & $6.7(3.8-12.1)$ \\
\hline Other high-risk types & 104 & (20) & 11 & (6) & 9 & (9) & $0.4(0.2-0.8)$ \\
\hline Possible high-risk types & 98 & (19) & 4 & $(2)$ & 4 & (4) & $0.2(0.1-0.6)$ \\
\hline Low-risk types & 214 & $(41)$ & 8 & (4) & 5 & (5) & $0.1(0.0-0.2)$ \\
\hline Multiple infections & 164 & $(31)$ & 16 & (9) & 6 & (6) & $0.2(0.1-0.4)$ \\
\hline
\end{tabular}

${ }^{\mathrm{a}} \mathrm{HPV}$ tested in cervical cells from women diagnosed with normal cytology from general population survey.

${ }^{\mathrm{b}} \mathrm{HPV}$ tested in biopsies from women diagnosed with histologically confirmed CIN3 and ICC from case series. 
increase for non-vaccine-targeted than vaccine-targeted types is likely because the former have more mismatches with both GP-PCR primers [5].

This baseline survey represents the first part of a longterm protocol to monitor HPV vaccine impact in Bhutan, which will include a repeat HPV prevalence survey in cervicovaginal samples in 2016/17 among women aged $\leq 24$ years to demonstrate the 5-year impact of the HPV vaccination programme, as well as repeated HPV prevalence surveys in urine samples of adolescents aged 17-19 years at highschools in Thimphu, Bhutan.

\section{Conclusions}

These data will serve as a robust baseline for future evaluations of HPV vaccine programme effectiveness on HPV prevalence as cohorts of vaccinated girls grow up. Nevertheless, our survey did also capture a few young vaccinated women in the baseline survey, among whom there was a very reassuring, albeit statistically weak, evidence of $100 \%$ protection against vaccine-targeted HPV type (HPV6/11/16/18) infection, irrespective of the HPV test. Whilst awaiting more statistically robust evaluations in the future, this ad hoc finding represents the first ever real-life evidence of HPV vaccination programme impact in a lowor middle-income country.

\section{Abbreviations \\ ASCUS/ASC-H/AGUS: Atypical squamous cells of undetermined significance, atypical squamous cells cannot exclude high-grade lesion or atypical glandular cells of undetermined significance; Cl: Confidence interval; CIN: Cervical intraepithelial neoplasia; HR: High-risk; HPV: Human papillomavirus; HSIL: High-grade squamous intraepithelial lesions; IARC: International Agency for Research on Cancer; ICC: Invasive cervical cancer; JDWNRH: Jigme Dorji Wangchuck National Referral Hospital; LMIC: Low- and middle-income country; LR: Low-risk; LSIL: Low-grade squamous intraepithelial lesions; OR: Odds ratio; PR: Prevalence ratio.}

\section{Competing interests}

P.J.F. Snijders has Honoraria from Speakers Bureau from Roche and is a consultant/advisory board member for Roche and Gen-Probe. C.J.L.M. Meijer has Honoraria of Speakers Bureau from GlaxoSmithKline and is a consultant/advisory board member for Qiagen. No competing interests were disclosed by the other authors.

\section{Authors' contributions \\ GC and SF conceived and designed the study. GC, SF, IB, UT and DD were involved in the development of the methodology. DD (cytology), PJFS (HPV testing), CJLMM and MCGB (histological review), TG and MT (HPV testing) collected data. GC, IB, SF, and VT performed data analysis and interpretation. All authors read and approved the final manuscript. DD was responsible for administrative, technical, or material support. GC supervised the study.}

\section{Acknowledgements}

The primary support for this project came from the International Agency for Research on Cancer and grants from the Bill \& Melinda Gates Foundation, USA (grant numbers 35537 and OPP1053353). The funding sources did not have any role in the writing process of this manuscript.

We would like to acknowledge the help of the following staff at JDWNR Hospital in Thimphu, Bhutan: Dr. Cheni Zangmo for colposcopy, Dr Tshokey for cytology and logistical support, Drs. I.K. Mohanta and B.M. Dhungel for cytology and histopathology, as well as Kinley Wangchuk and Samten for data entry. We are also grateful to the gynaecological and reproductive health nurses at JDWNRH and Lungtenphu hospitals, cytotechnicians at JDWNRH, and histotechnicians at JDWNRH and Mongar hospitals. We thank Veronique Chabanis for administrative assistance.

\section{Author details}

${ }^{1}$ Department of Obstetrics \& Gynaecology, Jigme Dorji Wangchuck National Referral Hospital, Thimphu, Bhutan. ${ }^{2}$ International Agency for Research on Cancer, 150 cours Albert Thomas, 69372 Lyon, cedex 08, France.

${ }^{3}$ Department of Laboratory Services, Jigme Dorji Wangchuck National Referral Hospital, Thimphu, Bhutan. ${ }^{4}$ VU University Medical Center, De Boelelaan 1117, 1081 HV Amsterdam, the Netherlands.

Received: 20 March 2014 Accepted: 12 July 2014

Published: 22 July 2014

\section{References}

1. Ferlay J, Soerjomataram I, Ervik M, Dikshit R, Eser S, Mathers C, Rebelo M, Parkin DM, Forman D, Bray F: GLOBOCAN 2012 v1.0, Cancer Incidence and Mortality Worlwide: IARC CancerBase No. 11 [Internet]. Lyon, France: International Agency for Research on Cancer; 2013.

2. Steward BW, Wild CP (Eds): World Cancer Report 2014. Lyon, France: International Agency for Research on Cancer; 2014

3. Clifford GM, Gallus S, Herrero R, Muñoz N, Snijders PJ, Vaccarella S, Anh PT, Ferreccio C, Hieu NT, Matos E, Molano M, Rajkumar R, Ronco G, de Sanjose S, Shin HR, Sukvirach S, Thomas JO, Tunsakul S, Meijer CJ, Franceschi S, and the IARC HPV Prevalence Surveys Study Group: Worldwide distribution of human papillomavirus types in cytologically normal women in the International Agency for Research on Cancer HPV prevalence surveys: a pooled analysis. Lancet 2005, 366:991-998.

4. Solomon D, Davey D, Kurman R, Moriarty A, O'Connor D, Prey M, Raab S, Sherman M, Wilbur D, Wright T Jr, Young N: The 2001 Bethesda System: terminology for reporting results of cervical cytology. JAMA 2002, 287:2114-2119.

5. Roda Husman AM, Snijders PJ, Stel HV, van den Brule AJ, Meijer CJ, Walboomers JM: Processing of long-stored archival cervical smears for human papillomavirus detection by the polymerase chain reaction. Br J Cancer 1995, 72:412-417.

6. Jacobs MV, Walboomers JM, Snijders PJ, Voorhorst FJ, Verheijen RH, Fransen-Daalmeijer N, Meijer CJ: Distribution of 37 mucosotropic HPV types in women with cytologically normal cervical smears: the age-related patterns for high-risk and low-risk types. Int I Cancer 2000, 87:221-227.

7. van den Brule AJ, Pol R, Fransen-Daalmeijer N, Schouls LM, Meijer CJ, Snijders PJ: GP5+/6+ PCR followed by reverse line blot analysis enables rapid and high-throughput identification of human papillomavirus genotypes. J Clin Microbiol 2002, 40:779-787.

8. IARC: Monographs on the Evaluation of Carcinogenic Risks to Humans Volume 90: Human Papillomaviruses [90]. Lyon: International Agency for Research on Cancer. Monographs on the Evaluation of carcinogenic Risk to Humans; 2007:1-670.

9. Gheit T, Tommasino M: Detection of high-risk mucosal human papillomavirus DNA in human specimens by a novel and sensitive multiplex PCR method combined with DNA microarray. Methods Mol Biol 2011, 665:195-212.

10. Eklund C, Zhou T, Dillner J: Global proficiency study of human papillomavirus genotyping. J Clin Microbiol 2010, 48:4147-4155.

11. Gheit T, Landi S, Gemignani F, Snijders PJ, Vaccarella S, Franceschi S, Canzian F, Tommasino M: Development of a sensitive and specific assay combining multiplex PCR and DNA microarray primer extension to detect high-risk mucosal human papillomavirus types. J Clin Microbiol 2006, 44:2025-2031

12. Keita N, Clifford GM, Koulibaly M, Douno K, Kabba I, Haba M, Sylla BS, van Kemenade FJ, Snijders PJ, Meijer CJ, Franceschi S: HPV infection in women with and without cervical cancer in Conakry, Guinea. Br J Cancer 2009, 101:202-208.

13. Dondog B, Clifford GM, Vaccarella S, Waterboer T, Unurjargal D, Avirmed D, Enkhtuya S, Kommoss F, Wentzensen N, Snijders PJ, Meijer CJ, Franceschi S, Pawlita M: Human papillomavirus infection in Ulaanbaatar, Mongolia: a population-based study. Cancer Epidemiol Biomarkers Prev 2008, 17:1731-1738.

14. Thomas JO, Herrero R, Omigbodun AA, Ojemakinde K, Ajayi IO, Fawole A, Oladepo O, Smith JS, Arslan A, Muñoz N, Snijders PJ, Meijer CJ, Franceschi S: 
Prevalence of papillomavirus infection in women in Ibadan, Nigeria: a population-based study. Br J Cancer 2004, 90:638-645.

15. Aruhuri B, Tarivonda L, Tenet V, Sinha R, Snijders PJ, Clifford G, Pang J, McAdam M, Meijer CJ, Frazer IH, Franceschi S: Prevalence of Cervical Human Papillomavirus (HPV) Infection in Vanuatu. Cancer Prev Res (Phila) 2012, 5:746-753.

16. Franceschi S, Rajkumar R, Snijders PJF, Arslan A, Mahé C, Plummer M, Sankaranarayanan R, Cherian J, Meijer CJLM, Weiderpass E: Papillomavirus infection in rural women in southern India. Br J Cancer 2005, 92:601-606.

17. Dai M, Bao YP, Li N, Clifford GM, Vaccarella S, Snijders PJF, Huang RD, Sun LX, Meijer CJLM, Qiao YL, Franceschi S: Human papillomavirus infection in Shanxi Province, People's Republic of China: a population-based study. Br J Cancer 2006, 95:96-101.

18. Wu RF, Dai M, Qiao YL, Clifford GM, Liu ZH, Arslan A, Li N, Shi JF, Snijders PJ, Meijer CJ, Franceschi S: Human papillomavirus infection in women in Shenzhen City, People's Republic of China, a population typical of recent Chinese urbanisation. Int J Cancer 2007, 121:1306-1311.

19. Li N, Franceschi S, Howell-Jones R, Snijders PJ, Clifford GM: Human papillomavirus type distribution in 30,848 invasive cervical cancers worldwide: Variation by geographical region, histological type and year of publication. Int I Cancer 2011, 128:927-935.

20. Sherpa AT, Clifford GM, Vaccarella S, Shrestha S, Nygård M, Karki BS, Snijders PJ, Meijer CJ, Franceschi S: Human papillomavirus infection in women with and without cervical cancer in Nepal. Cancer Causes Control 2010 21:323-330

21. Maucort-Boulch D, Franceschi S, Plummer M: International correlation between human papillomavirus prevalence and cervical cancer incidence. Cancer Epidemiol Biomarkers Prev 2008, 17:717-720.

22. Guan P, Howell-Jones R, Li N, Bruni L, de Sanjose S, Franceschi S, Clifford GM: Human papillomavirus types in 115,789 HPV-positive women: a meta-analysis from cervical infection to cancer. Int J Cancer 2012, 131:2349-2359.

23. Vaccarella S, Herrero R, Dai M, Snijders PJ, Meijer CJ, Thomas JO, Hoang Anh PT, Ferreccio C, Matos E, Posso H, de Sanjose S, Shin HR, Sukvirach S, Lazcano-Ponce E, Ronco G, Rajkumar R, Qiao YL, Muñoz N, Franceschi S: Reproductive factors, oral contraceptive use, and human papillomavirus infection: pooled analysis of the IARC HPV prevalence surveys. Cancer Epidemiol Biomarkers Prev 2006, 15:2148-2153.

24. Vaccarella S, Franceschi S, Herrero R, Muñoz N, Snijders PJF, Clifford GM, Smith JS, Lazcano-Ponce E, Sukvirach S, Shin HR, de Sanjose S, Molano M, Matos E, Ferreccio C, Anh PTH, Thomas JO, Meijer CJLM, and the IARC HPV Prevalence Surveys Study Group: Sexual behavior, condom use and HPV: pooled analysis of the International Agency for Research on Cancer HPV Prevalence Surveys. Cancer Epidemiol Biomarkers Prev 2006, 15:326-333.

doi:10.1186/1471-2334-14-408

Cite this article as: Tshomo et al:: Human papillomavirus infection in Bhutan at the moment of implementation of a national HPV vaccination programme. BMC Infectious Diseases 2014 14:408.

\section{Submit your next manuscript to BioMed Central and take full advantage of:}

- Convenient online submission

- Thorough peer review

- No space constraints or color figure charges

- Immediate publication on acceptance

- Inclusion in PubMed, CAS, Scopus and Google Scholar

- Research which is freely available for redistribution

Submit your manuscript at www.biomedcentral.com/submit 